\title{
Correction to "Identifying the ErbB/MAPK Signaling Cascade as a Therapeutic Target in Canine Bladder Cancer"
}

In the above article [Cronise KE, Hernandez BG, Gustafson DL, and Duval DL (2019) Mol Pharmacol 96:36-46; DOI: https://doi.org/10.1124/mol.119.115808], the following funding information was omitted from the article:

This article was supported by the Pharmacology Shared Resource for the University of Colorado Cancer Center, which is funded by the National Institutes of Health National Cancer Institute [Grant P30 CA046934].

The HTML and PDF versions of the article have been corrected to include this information. The authors regret the omission of this information and any inconvenience it may have caused. 